Supporting Information

\title{
Quantitative Measurement of Spatial Effects of DNA Origami on Molecular Binding Reactions Detected using Atomic Force Microscopy
}

Ping Zhang, ${ }^{+, \neq, \|}$Fei Wang, ${ }^{\#, l}$ Wenjing Liu, ${ }^{\dagger, t, l}$ Xiuhai Mao, ${ }^{\#}$ Changchun Hao, ${ }^{\S}$ Yi Zhang, ${ }^{\dagger, \theta, *}$ Chunhai Fan, ${ }^{,}, \#$ Jun Hu, ${ }^{\theta, \dagger}$ Lihua Wang, ${ }^{\dagger, \theta, ", *}$ and Bin $\mathrm{Li}^{\dagger, \theta, *}$

†Division of Physical Biology \& Bioimaging Centre, Shanghai Synchrotron Radiation Facility, CAS Key Laboratory of Interfacial Physics and Technology, Shanghai Institute of Applied Physics, Chinese Academy of Sciences, Shanghai 201800, China

University of Chinese Academy of Sciences, Beijing 100049, China

$\S$ Laboratory of Biophysics and Biomedicine, College of Physics and Information Technology, Shaanxi Normal University, Xi'an 710062, China

\#School of Chemistry and Chemical Engineering, and Institute of Molecular Medicine, Renji Hospital, School of Medicine, Shanghai Jiao Tong University, Shanghai 200240, China

${ }^{\theta}$ Shanghai Advanced Research Institute, Chinese Academy of Sciences, Shanghai 201210, China

"Shanghai Key Laboratory of Green Chemistry and Chemical Processes, School of Chemistry and Molecular Engineering, East China Normal University, Shanghai, 200241, China

\section{Corresponding Author}

*E-mail: libin@sinap.ac.cn; wanglihua@sinap.ac.cn; zhangyi@sinap.ac.cn 
Table S1. Nucleotide sequences of seven biotinylated staple strands and biotin sites modified on the triangular DNA origami.

\begin{tabular}{|c|c|l|}
\hline $\begin{array}{c}\text { Site } \\
\text { number }\end{array}$ & $\begin{array}{c}\text { Staple } \\
\text { strand }\end{array}$ & \multicolumn{1}{c|}{ Sequence $\left(5^{\prime} \rightarrow 3^{\prime}\right)$} \\
\hline 3 & A60-5' & biotin-TTCTGACCTAAAATATAAAGTACCGACTGCAGAAC \\
\hline 7 & B34-3' & CAACAGTTTATGGGATTTTGCTAATCAAAAGG-biotin \\
\hline 1 & B46-5' & biotin-AGGTTTAGTACCGCCATGAGTTTCGTCACCAGGATCTAAA \\
\hline 4 & B64-5' & $\begin{array}{l}\text { biotin- } \\
\text { ACCAACCTAAAAAATCAACGTAACAAATAAATTGGGCTTGAGA }\end{array}$ \\
\hline 6 & C13-5' & Biotin-GATTATACACAGAAATAAAGAAATACCAAGTTACAAAATC \\
\hline 2 & C54-5' & biotin-CGGCGGATTGAATTCAGGCTGCGCAACGGGGGATG \\
\hline 5 & C65-5' & $\begin{array}{l}\text { biotin- } \\
\text { ACGTGGACTCCAACGTCAAAGGGCGAATTTGGAACAAGAGTCC }\end{array}$ \\
\hline
\end{tabular}

Table S2. Additional nucleotide sequences of four biotinylated staple strands and biotin sites modified on the triangular DNA origami in supporting information.

\begin{tabular}{|c|c|c|}
\hline $\begin{array}{c}\text { Site } \\
\text { number }\end{array}$ & $\begin{array}{l}\text { Staple } \\
\text { strand }\end{array}$ & Sequence $\left(5^{\prime} \rightarrow 3^{\prime}\right)$ \\
\hline 8 & A39-5 & biotin-TTATCAAACCGGCTTAGGTTGGGTAAGCCTGT \\
\hline 9 & B23-5' & biotin- AACACT ATCATAACCCATCAAAAATCAGGTCTCCTTTTGA \\
\hline 10 & C45-5 & biotin- TTAATAAAACGAACTAACCGAACTGACCAACTCCTGATAA \\
\hline 11 & A08-5 & biotin-GACGGGAGAATT AACTCGGAATAAGTTTATTTCCAGCGCC \\
\hline
\end{tabular}

Table S3. Nucleotide sequences of 16 digoxin-labelled fifteen staple strands and digoxin sites modified on the rectangular DNA origami.

\begin{tabular}{|c|c|c|}
\hline $\begin{array}{c}\text { Site } \\
\text { number }\end{array}$ & $\begin{array}{l}\text { Staple } \\
\text { strand }\end{array}$ & Sequence $\left(5^{\prime} \rightarrow 3^{\prime}\right)$ \\
\hline \multirow{7}{*}{ rim } & $28-\mathrm{T}$ & CTCAGAGCCACCACCT-digoxin-TCATTTTCCT ATTATT \\
\hline & $51-5^{\prime}$ & digoxin-CCGCCAGCCATTGCAACAGGAAAAATATTTTT \\
\hline & $52-5^{\prime}$ & digoxin-CCCTCAGAACCGCCACCCTCAGAACTGAGACT \\
\hline & $99-5^{\prime}$ & digoxin-GAAATGGATTATTTACATTGGCAGACATTCTG \\
\hline & $133-5^{\prime}-\mathrm{T}$ & digoxin-CCCCGATTTAGAGCTT-digoxin-GACGGGGAAATCAAAA \\
\hline & $156-5^{\prime}$ & digoxin-TGTAGCATTCCACAGACAGCCCTCATCTCCAA \\
\hline & $180-5^{\prime}$ & digoxin-CGTAACGATCTAAAGTTTTGTCGTGAATTGCG \\
\hline \multirow{8}{*}{ surface } & $35-\mathrm{T}$ & GAAGGAAAATAAGAGT-digoxin-AAGAAACAACAGCCAT \\
\hline & $43-5^{\prime}$ & digoxin-ATCAAAATCGTCGCTA TTAATTAACGGATTCG \\
\hline & $60-5^{\prime}$ & digoxin-ATCAGAGAAAGAACT GGCATGATTTTATTTTG \\
\hline & $91-5^{\prime}$ & digoxin-TATGTAAACCTTTTTTAATGGAAAAATTACCT \\
\hline & $140-\mathrm{T}$ & AAATAATTTTAAATTT-digoxin-TAAACGTTGATATTCA \\
\hline & $141-5^{\prime}$ & digoxin-GCAAATATCGCGTCTGGCCTTCCTGGCCTCAG \\
\hline & $148-5^{\prime}$ & digoxin-CAAAATATAATGCAGATACATAAACACCAGA \\
\hline & $172-5^{\prime}$ & digoxin-TTTGCCAGATCAGTTGAGATTTAGTGGTTTAA \\
\hline
\end{tabular}


Table S4. Nucleotide sequences of 10 biotinylated staple strands and biotin sites modified on the rectangular DNA origami.

\begin{tabular}{|c|c|l|}
\hline $\begin{array}{c}\text { Site } \\
\text { number }\end{array}$ & $\begin{array}{c}\text { Staple } \\
\text { strand }\end{array}$ & \multicolumn{1}{c|}{ Sequence $\left(5^{\prime} \rightarrow 3^{\prime}\right)$} \\
\hline \multirow{4}{*}{ vertex } & $216-3^{\prime}$ & $\begin{array}{l}\text { CAGCGAAA TTTT AACTTTCA ACAGTTTCTGGGATTTTGCTAAAC } \\
\text { TTTT-biotin }\end{array}$ \\
\cline { 2 - 3 } & $111-3^{\prime}$ & $\begin{array}{l}\text { AAACCCTC TTTT ACCAGTAA TAAAAGGGATTCACCA GTCACACG } \\
\text { TTTT-biotin }\end{array}$ \\
\hline \multirow{3}{*}{ rim } & $156-5^{\prime}$ & biotin-TGTAGCATTCCACAGA CAGCCCTCATCTCCAA \\
\cline { 2 - 3 } & $28-5^{\prime}$ & biotin-CTCAGAGCCACCACCC TCATTTTC CTATTATT \\
\hline \multirow{4}{*}{ surface } & $150-3^{\prime}$ & ACGAGTAGTGACAAGA ACCGGATATACCAAGC-biotin \\
\cline { 2 - 3 } & $34-3^{\prime}$ & AGCGCCAACCATTTGG GAATTAGATTATTAGC-biotin \\
\cline { 2 - 3 } & $144-5^{\prime}$ & biotin-TCAATTCTTTTAGTTT GACCATTACCAGACCG \\
\cline { 2 - 3 } & $40-5^{\prime}$ & biotin- TAAGTCCTACCAAGTA CCGCACTC TTAGTTGC \\
\cline { 2 - 3 } & $116-5^{\prime}$ & biotin- TTCGCCAT TGCCGGAA ACCAGGCA TTAAATCA \\
\cline { 2 - 3 } & $20-5^{\prime}$ & biotin- CTTTTACACAGATGAA TATACAGT AAACAATT \\
\hline
\end{tabular}

Table S5. Nucleotide sequences of marker on the triangular DNA origami.

\begin{tabular}{|l|l|}
\hline IndexA02 & $\begin{array}{l}\text { AGCGTCATGTCTCTGATCCTCTTTTGAGGAACAAGTTTTCTTGTATTTACCG } \\
\text { ACTACCTT }\end{array}$ \\
\hline IndexA03 & $\begin{array}{l}\text { TTCATAATCCCCTTATTCCTCTTTTGAGGAACAAGTTTTCTTGTTAGCGTTTT } \\
\text { TCTTACC }\end{array}$ \\
\hline IndexA06 & $\begin{array}{l}\text { CCGGAACCCAGAATGGTCCTCTTTTGAGGAACAAGTTTTCTTGTAAAGCGCA } \\
\text { ACATGGCT }\end{array}$ \\
\hline IndexA07 & $\begin{array}{l}\text { AAAGACAACATTTTCGTCCTCTTTTGAGGAACAAGTTTTCTTGTGTCATAGC } \\
\text { CAAAATCA }\end{array}$ \\
\hline IndexA10 & $\begin{array}{l}\text { TGTACTGGAAATCCTCTCCTCTTTTGAGGAACAAGTTTTCTTGTATTAAAGCA } \\
\text { GAGCCAC }\end{array}$ \\
\hline IndexA11 & $\begin{array}{l}\text { CACCGGAAAGCGCGTTTCCTCTTTTGAGGAACAAGTTTTCTTGTTTCATCGGA } \\
\text { AGGGCGA }\end{array}$ \\
\hline
\end{tabular}

Table S6. Nucleotide sequences of marker on the rectangular DNA origami.

\begin{tabular}{|l|l|}
\hline Index 185 & $\begin{array}{l}\text { CTTGCATGCATTAATGTCCTCTTTTGAGGAACAAGTTTTCTTGTAATCGGCCCG } \\
\text { CCAGGG }\end{array}$ \\
\hline Index 187 & $\begin{array}{l}\text { TAGATGGGGGGTAACGTCCTCTTTTGAGGAACAAGTTTTCTTGTCCAGGGTTG } \\
\text { TGCCAAG }\end{array}$ \\
Index 189 & $\begin{array}{l}\text { CATGTCAAGATTCTCCTCCTCTTTTGAGGAACAAGTTTTCTTGTGTGGGAACC } \\
\text { GTTGGTG }\end{array}$ \\
\hline Index 191 & $\begin{array}{l}\text { CTGTAATATTGCCTGATCCTCTTTTGAGGAACAAGTTTTCTTGTGAGTCTGGAA } \\
\text { AACTAG }\end{array}$ \\
\hline Index206 & $\begin{array}{l}\text { TATCAGGGTTTCGGTTTTCCTCTTTTGAGGAACAAGTTTTCTTGTGCGTATTG } \\
\text { GGAACGCGCG }\end{array}$ \\
\hline Index207 & $\begin{array}{l}\text { GGGAGAGGTTTTGTAAATCCTCTTTTGAGGAACAAGTTTTCTTGTACGACGG } \\
\text { CCATTCCCAGT }\end{array}$ \\
\hline Index208 & $\begin{array}{l}\text { CACGACGTTTTGTAATGTCCTCTTTTGAGGAACAAGTTTTCTTGTGGATAGGT } \\
\text { CAAAACGGCG }\end{array}$ \\
\hline Index209 & $\begin{array}{l}\text { GATTGACCTTTTGATGAATCCTCTTTTGAGGAACAAGTTTTCTTGTCGGTAATC } \\
\text { GTAGCAAACA }\end{array}$ \\
\hline
\end{tabular}




\section{Results and Figures}

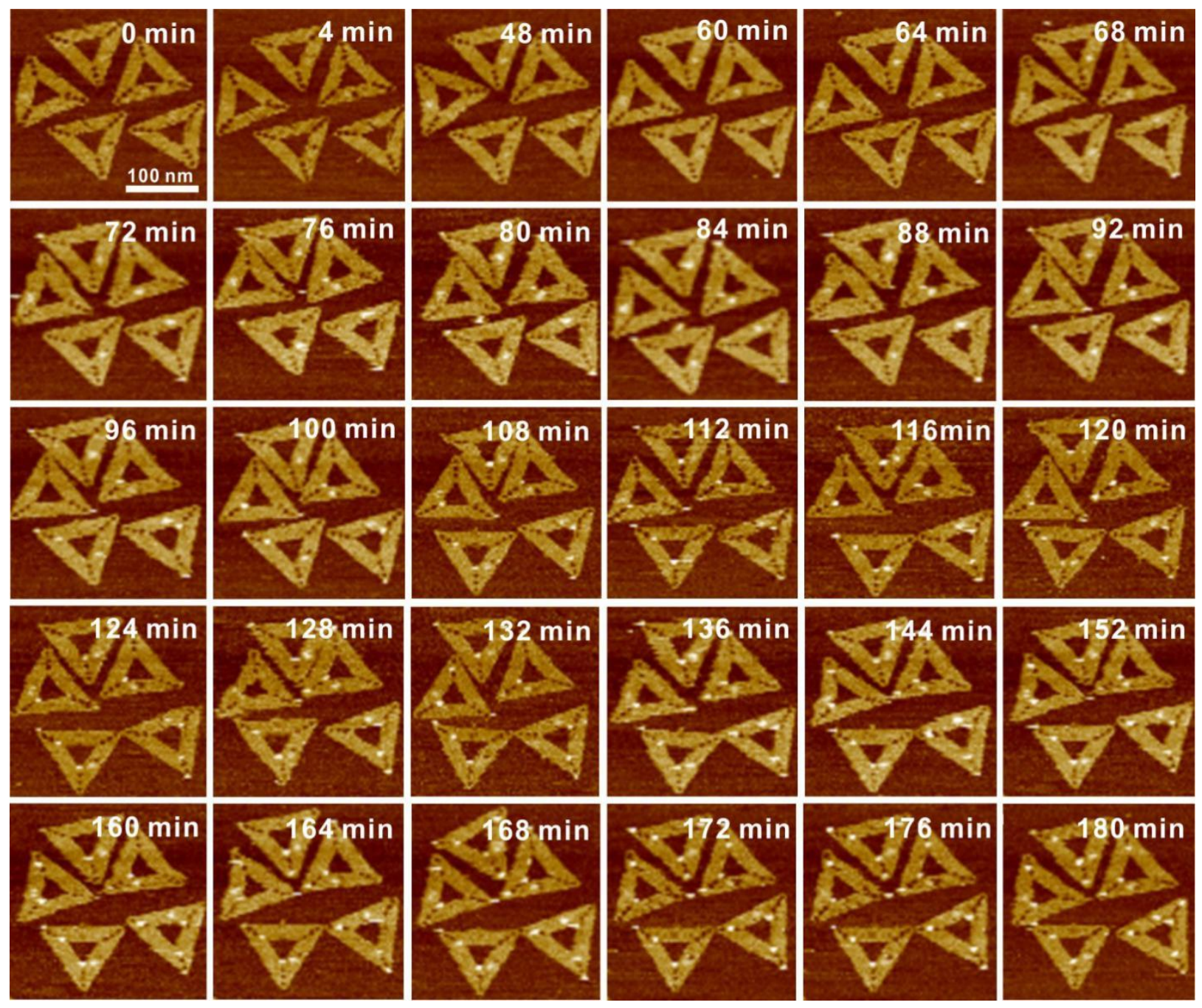

Fig. S1. Dynamic process of AFM images of biotin-SA complexes formed on the DNA origami surface when adsorbing on the mica surface for $180 \mathrm{~min}$. 

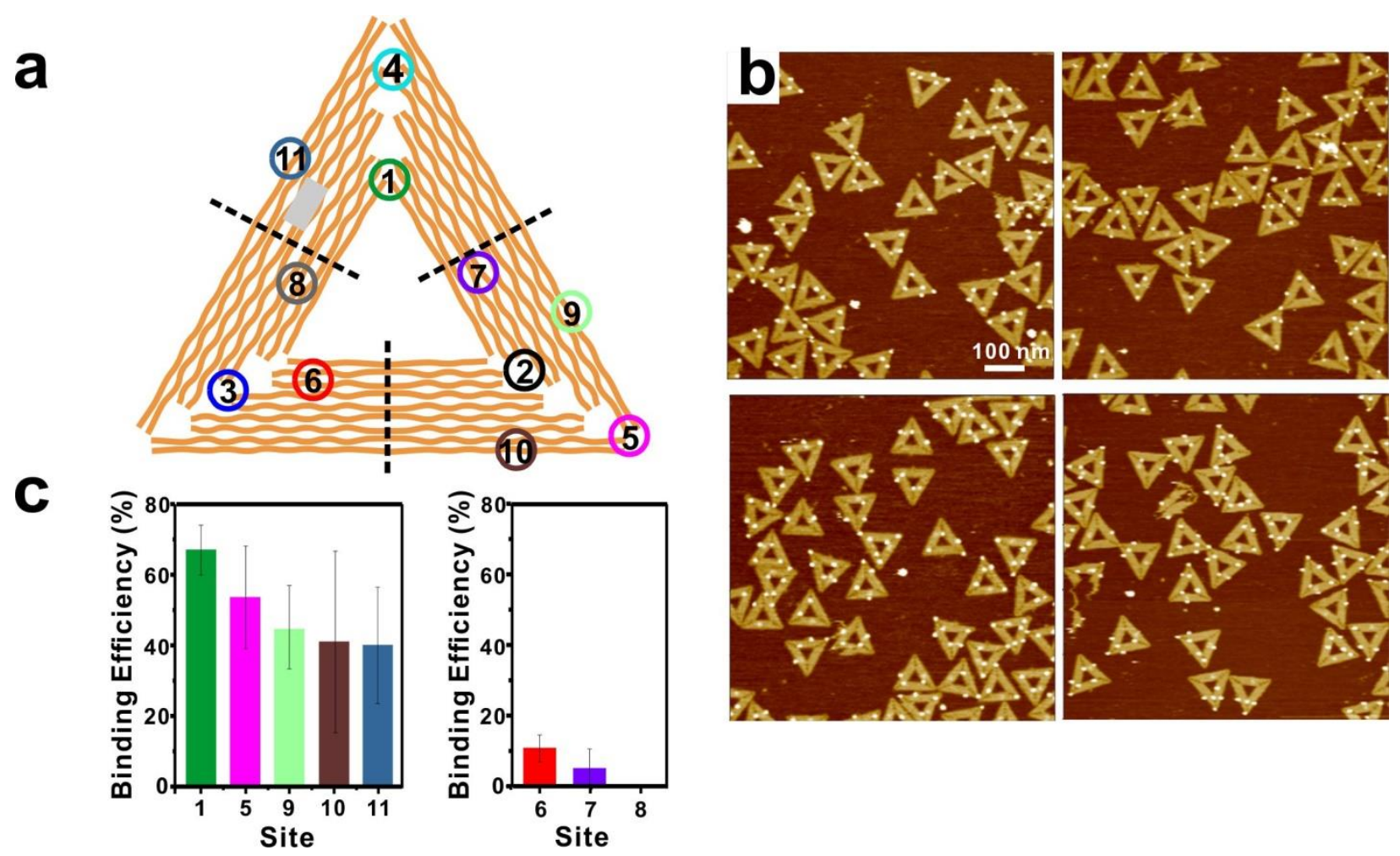

Fig. S2. Binding efficiency of SA to biotin molecules at different locations of the triangular DNA origami adsorbed on a mica surface. (a) Schematic representation of sites modified with biotin on the triangular DNA origami. (b) AFM images of biotin-SA complexes formed on the triangular DNA origami (c) Binding efficiency of the different biotinylated sites of DNA origami. The error bars presented standard deviations ( $\mathrm{n}=173$ DNA origamis). 

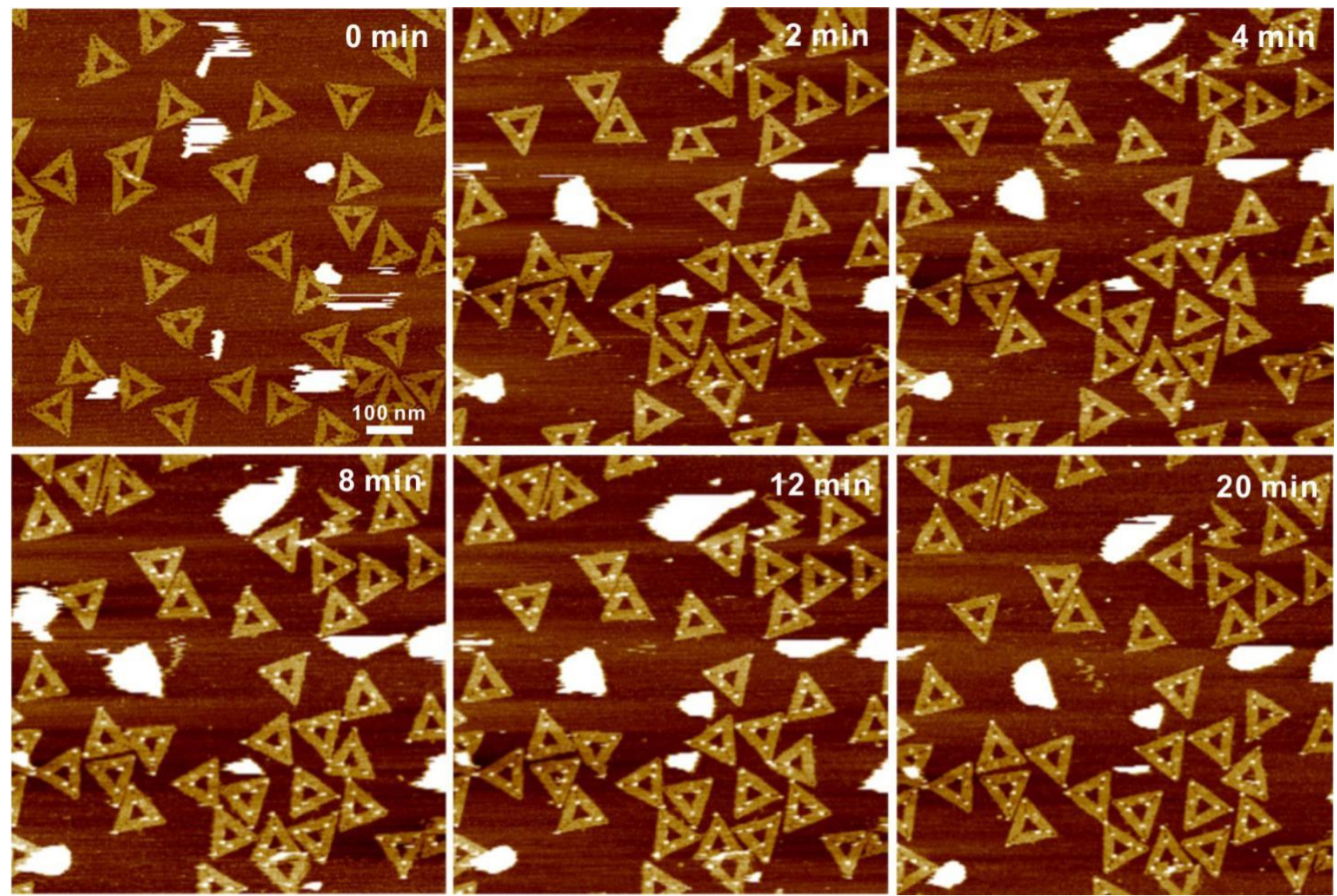

Fig. S3. AFM images for recording the biotin-SA formation for $20 \mathrm{~min}$ for the selected concentration of SA $(17.5 \mathrm{nM})$ when the DNA origamis adsorbing on mica surface. 

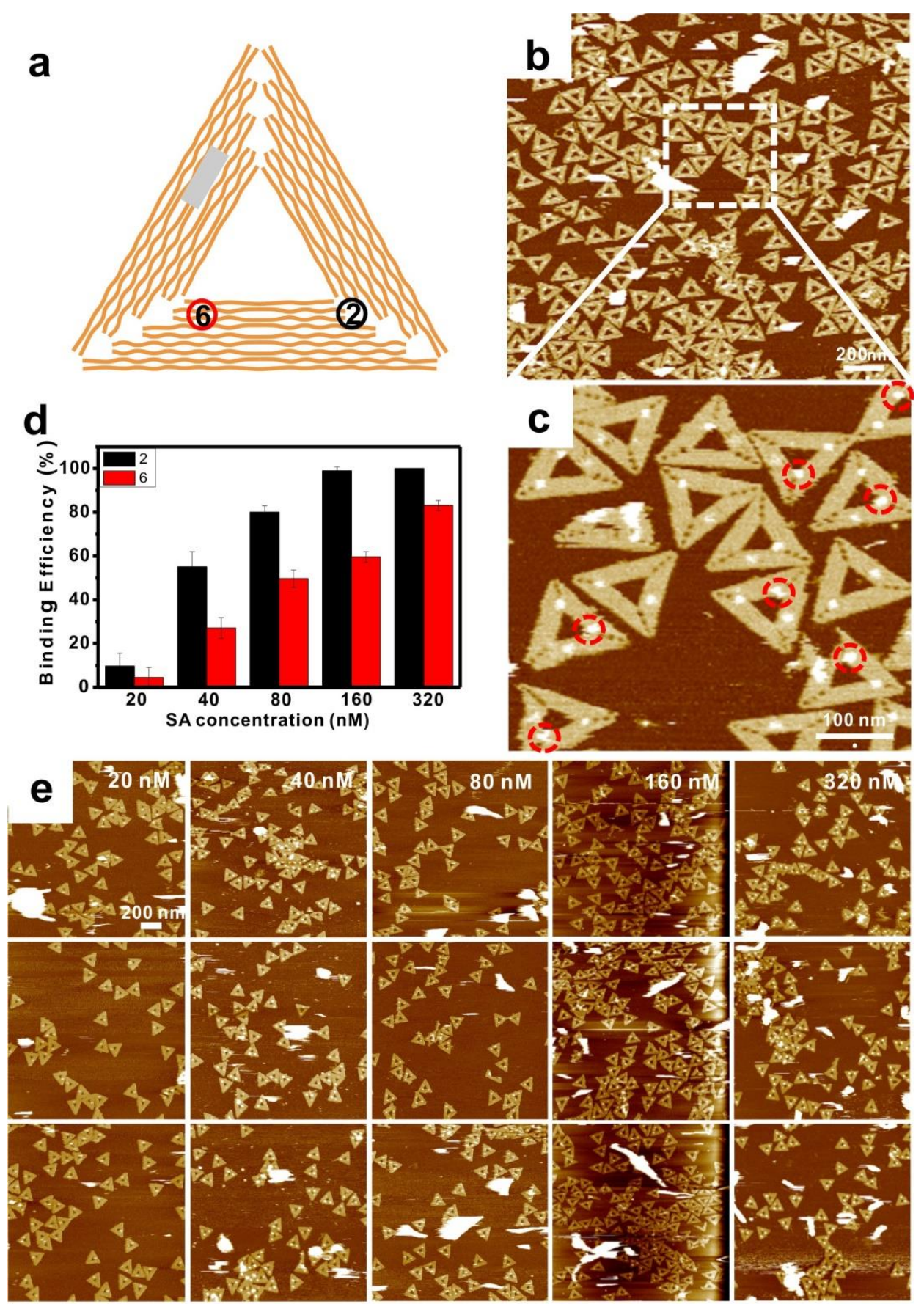

Fig. S4. Comparison of biotin-SA binding efficiency in solution between two sites: corner site 2 and surface site 6. (a) Outline of the biotin locations for sites 2 and 6 on the DNA origami. (b) AFM images of biotin-SA complexes on the DNA origami. (c) Enlarged image from the area in (b) marked by a white square. Binding SA on site 6 was marked with a red circle. (d) Binding efficiency of two sites as a function of SA concentration $(n=468$ DNA origamis $)$. (e) AFM images of the biotin-SA complexes formed in solution with SA concentrations of $20 \mathrm{nM}, 40 \mathrm{nM}, 80 \mathrm{nM}$, $160 \mathrm{nM}$ and $320 \mathrm{nM}$. 

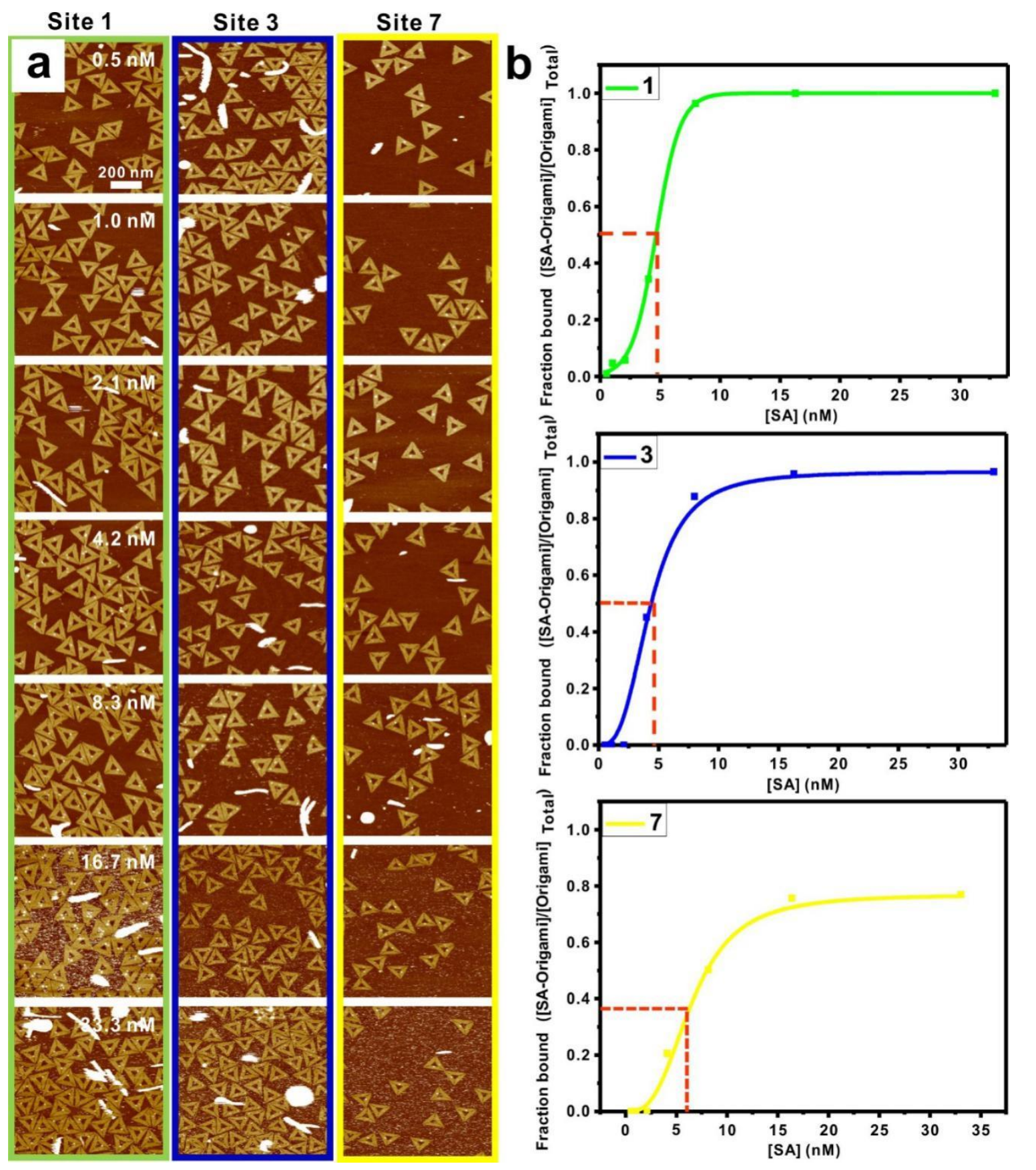

Fig. S5. Biotin-SA binding curve obtained from plotting the amount of biotin-SA complex as a function of free SA concentration at sites 1, 3 and 7, respectively. (a) AFM images of biotin-SA complexes. (b) Shown are the $K_{\mathrm{d}}$, the concentration of SA indicated by the dashed lines at which the fraction bound is 0.5 . 

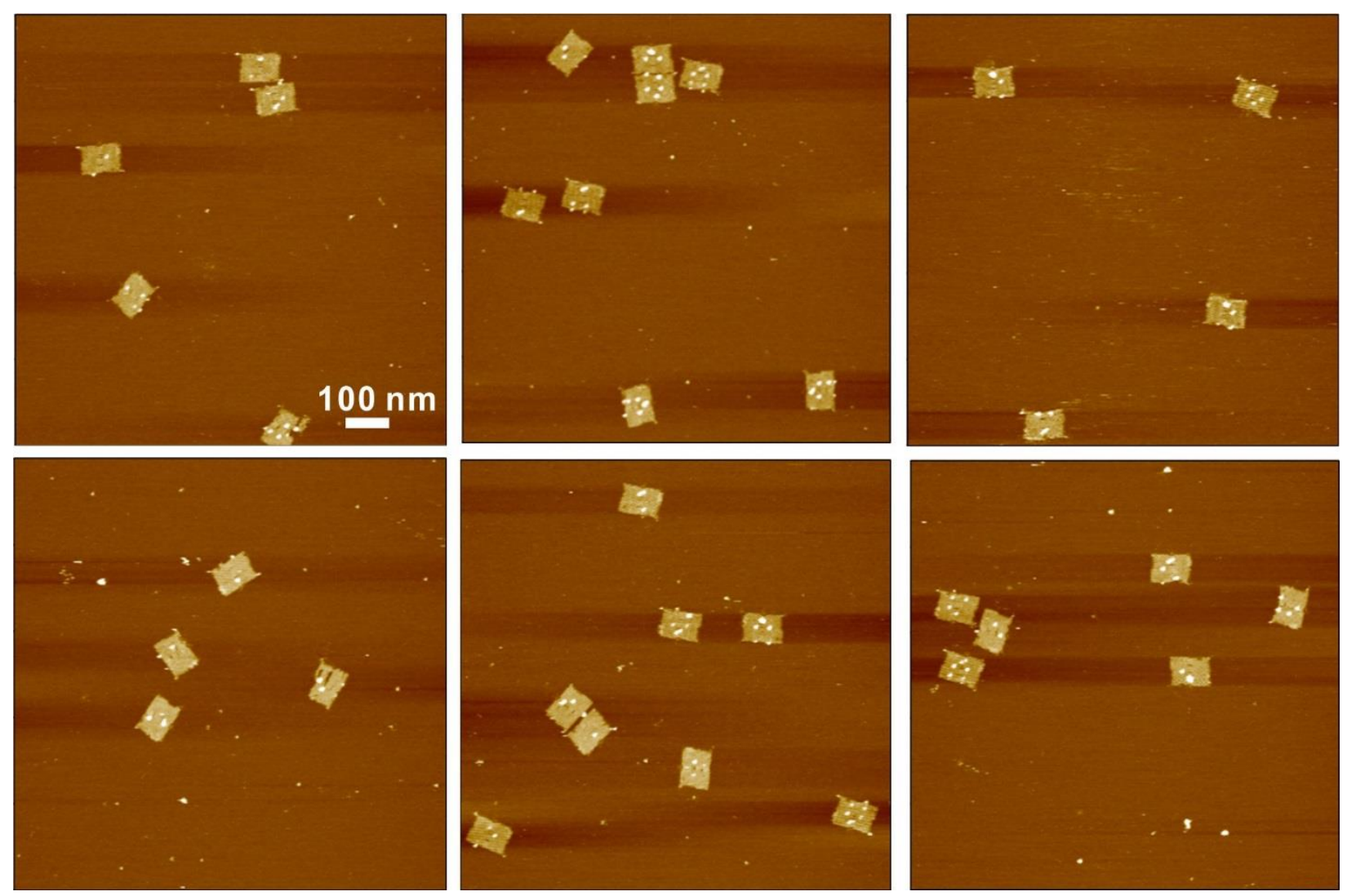

Fig. S6. AFM images of biotin-SA complexes formed on the rectangular DNA origami on a mica surface.
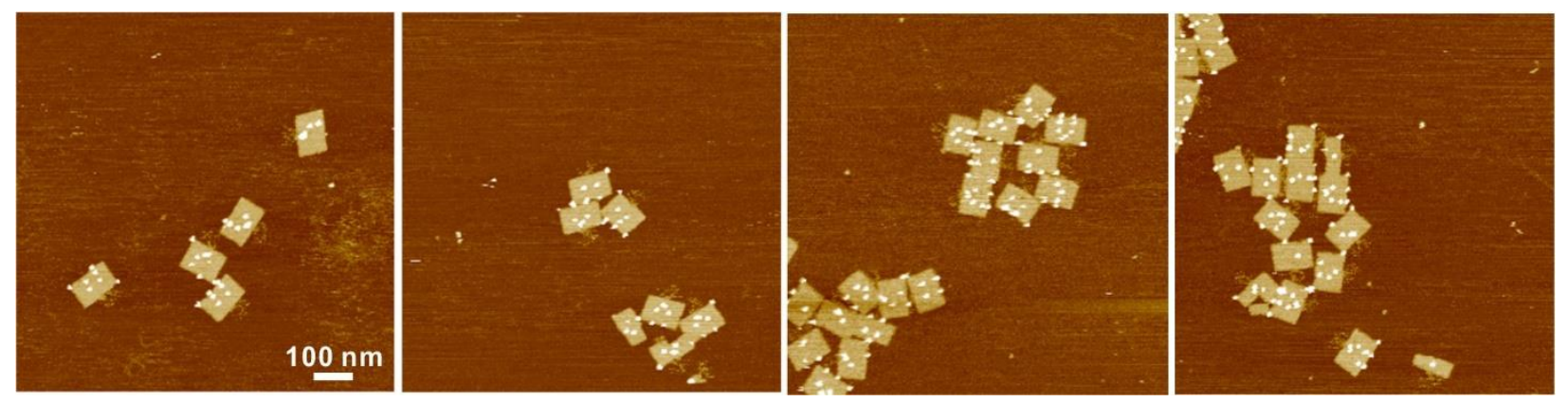

Fig. S7. AFM images of biotin-SA complexes formed on the rectangular DNA origami in solution. 

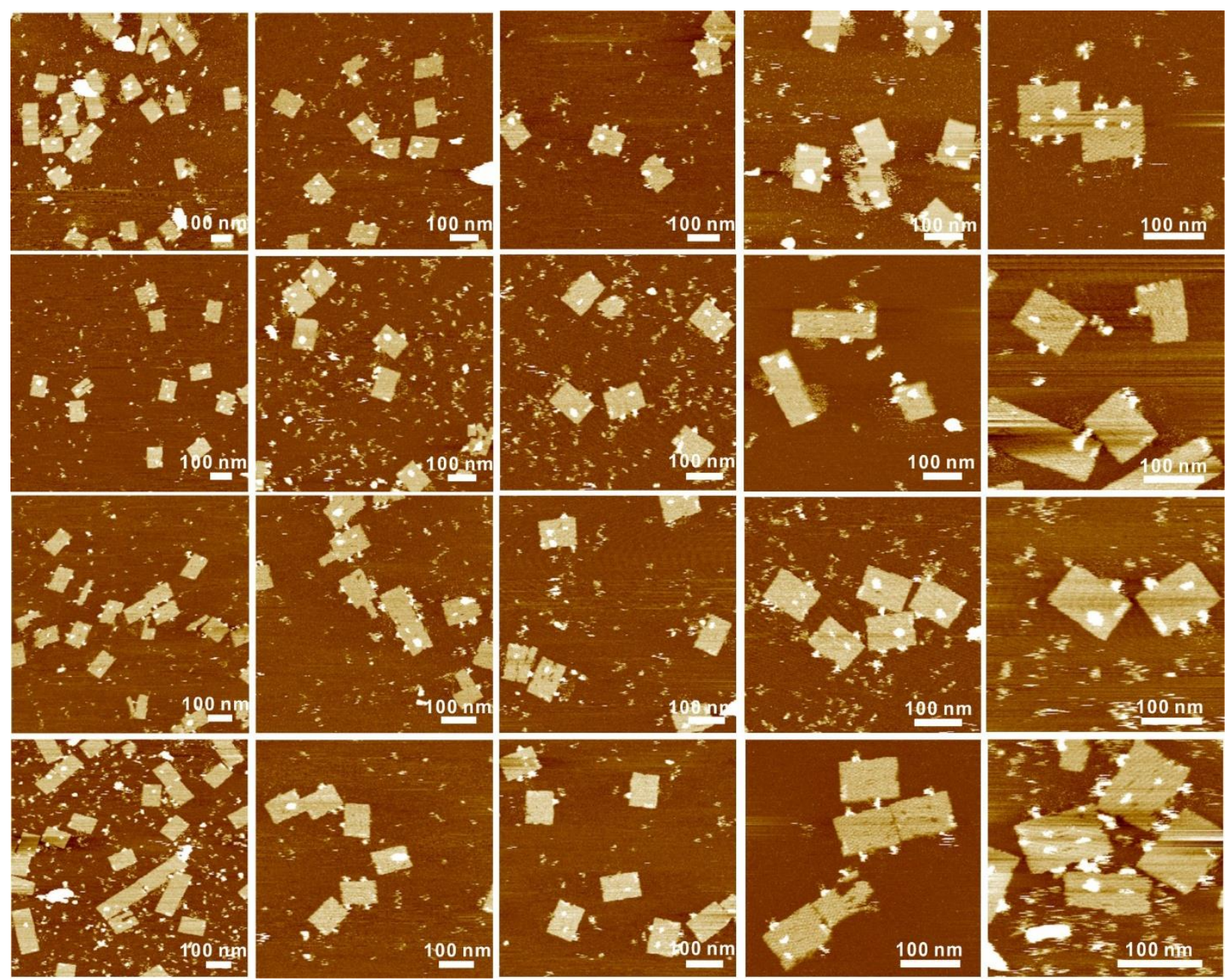

Fig. S8. AFM images of digoxin-IgG complexes formed on the DNA origami surface in solution. 